\title{
Investigation on Psychological Health and Resilience of Clinical Nursing Students in "Post-Pandemic Era"
}

\section{-A Case Study of Clinical Nursing Students in a Grade III Level A Hospital in Guangzhou}

\author{
Mei Zhang, Xiaoli Xin, Rong $\mathrm{Li}^{*}$ \\ The Third Affiliated Hospital of Sun Yat-sen University, Guangzhou, China \\ Email: *154083927@qq.com
}

How to cite this paper: Zhang, M., Xin, X.L. and Li, R. (2021) Investigation on Psychological Health and Resilience of Clinical Nursing Students in "Post-Pandemic Era". Open Journal of Medical Psychology, 10, 70-79.

https://doi.org/10.4236/ojmp.2021.104007

Received: August 30, 2021

Accepted: October 6, 2021

Published: October 9, 2021

Copyright $\odot 2021$ by author(s) and Scientific Research Publishing Inc. This work is licensed under the Creative Commons Attribution International License (CC BY 4.0).

http://creativecommons.org/licenses/by/4.0/

\begin{abstract}
The study aims to learn about the psychological health and counseling situation of nursing students in the post-pandemic era through investigation on the psychological health, resilience and counseling demand of nursing students by randomly selecting clinical nursing students interned in a Grade III Level A hospital in Guangzhou from June 2020 to January 2021 as the objects of study. The internship can be divided into three stages: initial (1st - 3rd month), middle (4th - 7th month) and final (8th - 12th month). The investigator educated the nursing students about psychological health in the initial stage to improve their adaptability to clinical practice. Then they were given questionnaires including Symptom Checklist 90 (SCL-90), Connor-Davidson Resilience Scale (CD-RISC) and general questionnaires. And this study further completed difference analysis on psychological health and resilience in gender, educational background, internship stages and non-only/only children. It turned out that all factors scored above the national norm except anxiety in nine symptom clusters and three dimensions of CD-RISC. As for the scores of four factors of somatization, interpersonal relationship, hostility and bigotry and three dimensions of tenacity, optimism and strength in the $\mathrm{CD}$-RISC, the difference was considered to indicate statistical significance $(\mathrm{P}$ $<0.001)$. Among factors affecting the psychological health of clinical nursing students, score differences involving somatization and compulsion $(\mathrm{P}<0.001$, $\mathrm{P}<0.05)$ under different educational backgrounds showed statistically significant. With regard to different clinical stages, there were significant differences in somatization, compulsion, tenacity and strength $(\mathrm{P}<0.001, \mathrm{P}<$
\end{abstract}


0.05). Finally, the conclusion is drawn that, during the period of pandemic prevention and control, the overall psychological status of clinical nursing students was good, but there remain part of cases with mild psychological problems that cannot be ignored. In the post-pandemic era, it is necessary to strengthen the awareness of teaching groups of clinical hospitals to psychological counseling for nursing students.

\section{Keywords}

Post-Pandemic Era, Clinical Nursing Students, Psychological Health, Psychological Resilience

\section{Introduction}

Nursing interns, as a backup reserve for future nursing work, must not only master solid professional knowledge but also maintain a healthy mental state, which is vital to the future development of the motherland's medical career. Psychological resilience, as a kind of good adaptability that an individual realizes in life crisis events, has positive significance for the individual's physical and mental health. Wu Man conducts research on the mental health of nurses. Better mental flexibility can effectively alleviate the negative effects of anxiety and depression and improve the mental health of nurses.

All industries and schools in China have entered the "post-pandemic era" with the widespread vaccination and further controlled pandemic at home and abroad. Infectious pandemic indicates an important stressor that causes individual physiological and psychological changes [1]. Once the inner balance is broken, it comes to fear, anxiety, depression, insomnia and other physiological and psychological responses, which will develop into PTSD, depression and even self-injury and suicide without timely intervention [2]. The study shows that special groups, including infected patients, front-line medical staff, and pandemic prevention personnel in communities, and the general public have high incidence of anxiety, depression and PTSD [3] [4]. Hospitals are at the forefront of the anti-virus battle that confronts more pressure and challenges. Those would-be nursing students never had such an experience testing both physical and mental endurance. Except for being exposed to academic pressure, they have to cope with psychological impacts brought by changes in learning method, living environment, interpersonal communication and closer proximity to virus. There are some problems to be settled with important management tasks of nursing students in hospitals concerning health examination before resumption, self-prevention and control after resumption, emerging psychological pressure and heavy teaching tasks. The changes and impacts of the pandemic on nursing students' psychological health are the most important but least heeded. Therefore, it is of great significance for flexible and active countermeasures against psychological health 
management by teaching groups, and dynamic integration of teaching activities with regular pandemic prevention and control [5] [6]. Psychological resilience, as a kind of good adaptability that an individual realizes in life crisis events, has positive significance for the individual's physical and mental health. Wu Man conducts research on the mental health of nurses. Better mental flexibility can effectively alleviate the negative effects of anxiety and depression and improve the mental health of nurses [7]. This study investigated the change of psychological health and resilience of clinical nursing students interned in a Grade III Level A hospital in Guangzhou from June 2020 to January 2021 in the "post-pandemic era", and analyzed relevant influencing factors to provide a reference for subsequent targeted psychological prevention and crisis intervention.

\section{Objects and Methods}

\subsection{Objects}

This study took clinical nursing students interned in a Grade III Level A hospital in Guangzhou from June 2020 to January 2021 as the objects. Inclusion criteria: 1) full-time nursing students serving as clinical interns; 2) satisfactory completion of school courses; 3) voluntary participation in this study. Exclusion criteria: nursing students suffering from mental and psychological disorders. Removal criteria: 1) nursing students suspending or terminating the internship due to personal reasons; 2) nursing interns in probation, clinical learning and other non-clinical stages. A total of 143 questionnaires were distributed and collected completely during the investigation.

\subsection{Methods}

Nursing students undergo a one-week pre-job training before the internship. The content mainly includes hospital culture, system, communication, and mental health. According to the principle of voluntariness, clinical nursing students are invited to fill out questionnaires on mental health status and mental resilience from the three dimensions of basic data, SCL-90, and CD-RISC.

Tools used for the investigation include SCL-90 and CD-RISC (Chinese version). 1) SCL-90 [8] was used to assess the psychological health of nursing students, including nine symptom factors: somatization, compulsion, interpersonal relationship, depression, anxiety, hostility, bigotry, psychosis in five grades: none, mild, moderate, moderately severe and severe recorded as 1, 2, 3, 4 and 5 respectively. The higher the value, the more serious the symptoms are. Shi Lingyan, et al. [9] verified that the SCL-90 possesses good reliability and validity based on the $\alpha$ value of 0.954 . 2) The Chinese version of CD-RISC was translated by two Chinese scholars Yu Xiaonan and Zhang Jianxin [10] [11]. The original CD-RISC was co-produced by professors Connor and Davidson [12] from Duke University, the USA. It is mainly used to measure the positive adaptability of individuals in adversity. The Chinese version of CD-RISC contains 25 items in three dimensions of tenacity (13), strength (8) and optimism (4). The applied Likert 5-level 
scale has scores ranging from 0 to 4 , and the total score ranges from 0 to 100 , with a higher score indicating the higher psychological resilience. The $\alpha$ coefficient was finally calculated to be 0.91 showing good reliability and validity. 3) Self-designed general data questionnaires, including gender, age, educational background and non-only/only children.

\subsection{Statistical Analysis}

The statistics software SPSS 22.0 was used for data analysis. The measurement data are expressed by $(\bar{x} \pm s)$ with $\mathrm{t}$ test, and the counting data comparison with chi-square test. The standard refers to $\alpha=0.05$.

\section{Results}

\subsection{General Conditions}

A total of 143 questionnaires were collected, with the average age of objects of $21.26 \pm 2.17$ years old. There are 5 males and 138 females, including 54 persons with junior college degree and 89 persons with full-time bachelor degree, and 16 only children and 127 non-only children (Table 1).

\subsection{Results of SCL-90 and CD-RISC}

1) Psychological health of nursing students: During the COVID-19 pandemic prevention and control, it has been turned out that all factors scored above the national norm except anxiety in nine symptom clusters and three dimensions of CD-RISC involving nursing students from a Grade III Level A hospital. As for the scores of four factors of somatization, interpersonal relationship, hostility and bigotry and three dimensions of tenacity, optimism and strength in the CD-RISC, the difference was considered to indicate statistical significance $(\mathrm{P}<$ 0.001) (Table 2, Table 3).

2) Comparison of influencing factors of SCL-90 and CD-RISC dimensions. With regard to different educational background, there were significant differences in somatization and compulsion $(\mathrm{P}<0.001, \mathrm{P}<0.05)$. With regard to different clinical stages, there were significant differences in somatization, compulsion, tenacity and strength $(\mathrm{P}<0.001, \mathrm{P}<0.05)$ (Table 4, Table 5).

Table 1. General data distribution $(\mathrm{n}=143)$.

\begin{tabular}{cccc}
\hline \multirow{2}{*}{ Item } & Classification & $\begin{array}{c}\text { Number of } \\
\text { People }\end{array}$ & $\begin{array}{c}\text { Percentage } \\
(\%)\end{array}$ \\
\hline \multirow{2}{*}{ Gender } & Male & 5 & 3.49 \\
& Female & 138 & 96.51 \\
\hline \multirow{2}{*}{ Educational background } & Junior college degree & 54 & 37.76 \\
& Bachelor degree & 89 & 62.24 \\
\hline \multirow{2}{*}{ Only children } & Yes & 16 & 11.18 \\
& No & 127 & 88.82 \\
\hline
\end{tabular}


Table 2. Comparison of SCL-90 result of nursing students and the national norm (Score, $\bar{x} \pm s)$.

\begin{tabular}{ccccc}
\hline Factor & Nursing Student & National Norm & T Value & P Value \\
\hline Somatization & $1.19 \pm 0.32$ & $1.37 \pm 0.48$ & -4.401 & $<0.001$ \\
Compulsion & $1.57 \pm 0.57$ & $1.62 \pm 0.58$ & -1.012 & 0.312 \\
Interpersonal Relationship & $1.46 \pm 0.11$ & $1.65 \pm 0.51$ & -4.479 & $<0.001$ \\
Depression & $1.47 \pm 0.10$ & $1.5 \pm 0.59$ & -0.653 & 0.514 \\
Anxiety & $1.41 \pm 0.09$ & $1.39 \pm 0.43$ & 0.555 & 0.579 \\
Hostility & $1.31 \pm 0.09$ & $1.48 \pm 0.56$ & -3.568 & $<0.001$ \\
Fear & $1.28 \pm 0.06$ & $1.23 \pm 0.41$ & 1.550 & 0.121 \\
Bigotry & $1.26 \pm 0.07$ & $1.43 \pm 0.57$ & -3.553 & $<0.001$ \\
Psychosis & $1.29 \pm 0.09$ & $1.29 \pm 0.42$ & 0.013 & 0.99 \\
\hline
\end{tabular}

Table 3. Comparison of CD-RISC result of nursing students and the national norm (Score, $\bar{x} \pm s)$.

\begin{tabular}{ccccc}
\hline Factor & Nursing Student & National Norm & T Value & P Value \\
\hline Tenacity & $2.42 \pm 0.09$ & $32.04 \pm 5.34$ & -66.320 & $<0.001$ \\
Optimism & $2.40 \pm 0.06$ & $8.05 \pm 3.01$ & -22.450 & $<0.001$ \\
Strength & $2.68 \pm 0.07$ & $21.34 \pm 6.28$ & -35.514 & $<0.001$ \\
Total average score & $8.6 \pm 2.67$ & $1.44 \pm 0.43$ & 89.591 & $<0.001$ \\
Total score & $61.43 \pm 17.95$ & $129.96 \pm 38.76$ & -20.909 & $<0.001$ \\
\hline
\end{tabular}

Table 4. Comparison of SCL-90 factors and CD-RISC results of nursing students in different clinical stages.

\begin{tabular}{ccccc}
\hline Factor & Initial Stage & Middle Stage & Final Stage & P Value \\
\hline Somatization & $1.09 \pm 0.17$ & $1.29 \pm 0.39$ & $1.14 \pm 0.19$ & 0.009 \\
Compulsion & $1.31 \pm 0.3$ & $1.77 \pm 0.66$ & $1.46 \pm 0.44$ & 0.000 \\
Interpersonal Relationship & $1.45 \pm 0.08$ & $1.47 \pm 0.14$ & $1.42 \pm 0.02$ & 0.188 \\
Depression & $1.47 \pm 0.09$ & $1.47 \pm 0.12$ & $1.43 \pm 0.04$ & 0.263 \\
Anxiety & $1.41 \pm 0.08$ & $1.41 \pm 0.11$ & $1.38 \pm 0.03$ & 0.409 \\
Hostility & $1.32 \pm 0.07$ & $1.31 \pm 0.11$ & $1.29 \pm 0.04$ & 0.516 \\
Fear & $1.28 \pm 0.06$ & $1.29 \pm 0.07$ & $1.27 \pm 0.02$ & 0.396 \\
Bigotry & $1.28 \pm 0.06$ & $1.26 \pm 0.09$ & $1.24 \pm 0.04$ & 0.166 \\
Psychosis & $1.29 \pm 0.06$ & $1.3 \pm 0.11$ & $1.27 \pm 0.03$ & 0.420 \\
Tenacity & $2.41 \pm 0.06$ & $2.41 \pm 0.11$ & $2.46 \pm 0.03$ & 0.083 \\
Optimism & $2.39 \pm 0.02$ & $2.41 \pm 0.07$ & $2.4 \pm 0.03$ & 0.165 \\
Strength & $2.68 \pm 0.04$ & $2.67 \pm 0.08$ & $2.72 \pm 0.03$ & 0.021 \\
\hline
\end{tabular}


Table 5. Comparison of SCL-90 factors and CD-RISC results under different educational backgrounds (Score, $\bar{x} \pm s$ ).

\begin{tabular}{cccc}
\hline Factor & Male & Female & P Value \\
\hline Somatization & $1.18 \pm 0.25$ & $1.19 \pm 0.32$ & 0.965 \\
Compulsion & $1.4 \pm 0.25$ & $1.57 \pm 0.58$ & 0.505 \\
Interpersonal Relationship & $1.48 \pm 0.11$ & $1.46 \pm 0.11$ & 0.709 \\
Depression & $1.49 \pm 0.13$ & $1.47 \pm 0.1$ & 0.564 \\
Anxiety & $1.43 \pm 0.11$ & $1.41 \pm 0.09$ & 0.629 \\
Hostility & $1.32 \pm 0.08$ & $1.31 \pm 0.09$ & 0.775 \\
Fear & $1.3 \pm 0.09$ & $1.28 \pm 0.06$ & 0.661 \\
Bigotry & $1.29 \pm 0.09$ & $1.26 \pm 0.07$ & 0.330 \\
Psychosis & $1.3 \pm 0.08$ & $1.29 \pm 0.09$ & 0.774 \\
Tenacity & $2.4 \pm 0.08$ & $2.42 \pm 0.09$ & 0.652 \\
Optimism & $2.38 \pm 0.05$ & $2.4 \pm 0.06$ & 0.532 \\
Strength & $2.67 \pm 0.06$ & $2.68 \pm 0.07$ & 0.684 \\
\hline
\end{tabular}

\section{Discussion}

\subsection{Analysis of Psychological Health of Clinical Nursing Students from SCL-90 and CD-RISC Results}

During the pandemic prevention and control, for a Grade III Level A hospital in Guangzhou, all factors scored above the national norm except anxiety in both SCL-90 and three dimensions of CD-RISC. The reasons are as follow based on analysis. 1) Overwhelming pandemic and daily news caused social panic. High infectivity and pathogenicity of COVID-19 put enormous pressure on clinical nurses, even if they, particularly new nursing students, worked with great professionalism and inner strength, and affected their psychological health seriously as well; 2) For being pressed by internship and employment, the internship progress was delayed under such pandemic. Many courses shifted from offline to online. Teaching methods were changed. And the health qualification examination was postponed. Many clinical nursing students have borne strong psychological pressure when dealing with qualification examination preparation, graduation paper as well as heavy tasks in clinical internship; 3) The living environment was changed. Many nursing students left their hometown and familiar environment, and had not adapted them from school students to clinical nursing students. Besides learning theoretical knowledge and operational skills, they were required to communicate with patients, family members and teachers every day. They came up against a great deal of resistance in interpersonal communication. The investigation found that most nursing students' biggest concern and stress are from communication. Some students said that they had no idea about how to carry out health education for patients and their family members, tones as well as methods? During the pandemic prevention and control, family members were not allowed to accompany patients which brought great difficulties to 
clinical management. Students had to communicate and explain with patients and their family members every day. They will be perplexed on explanation to picky family members of patients. Those post-1995s and post-2000s are generally concerned and tolerated at home. However, they met people with different personalities and backgrounds. Appropriate communicate became the big difficulty. All above matters may cause the anxiety factor of nursing students to be higher than the norm. However, according to the investigation, other factors of SCL-90 and three dimensions of CD-RISC scored lower than the norm, which may be closely related to the psychological health lectures prior to the internship. Such lectures focused on four aspects including common clinical psychological problems, emotion processing, emotion self-adjustment and peer interaction. After the targeted orientation, students had a sense on the environment, culture and nursing team of the internship hospital, and knew and adapted quickly to the new environment. Their sense of unfamiliarity and anxiety on the hospital and work were eliminated, which made they adapt to the role fast. Psychological construction training should be carried out against common clinical internship problems concerning communication, operation and learning. Students should be taught about how to deal with emotion and adjust themselves with mindfulness. Finally, nursing students were required to discuss on the following three aspects: 1) What makes you most stressed about the upcoming clinical internship? 2) How to cope with the possible upcoming pressure? 3) What kind of nurse do you want to be? Psychological health problems of nursing students during the pandemic should be given advanced focus and support. And psychological health also relates to a better psychological quality. According to the study of Xin Ziqiang, et al. [13], psychological health of college students, particularly involving interpersonal sensitivity, depression and hostility, has been improved year by year.

\subsection{Comparison of Influencing Factors of Psychological Health}

With regard to different educational background, there were significant differences in somatization and compulsion $(\mathrm{P}<0.001, \mathrm{P}<0.05)$. For undergraduates, their results of psychological health are lower than the national norm but higher than those of junior college students. They have strong learning and logical thinking abilities, and got college admission scores much closer to the major of Clinical Medicine and are positive and motivated, they are similar to those of clinical medical students in college entrance examination, and they are positive and motivated, sensitive in thinking, indomitable and striving for perfection. On the contrary, junior college students have low education backgrounds, are vulnerable and sensitive, and have a poor compression ability, resulting in psychological imbalance easily. As a result, the undergraduates have a better psychological health than junior college students. Liu Yuanyuan [14] found that people with higher educational background have a better psychological status. With strong practical and communication skills, junior college students work hard and are eager to learn, and accept temporary backwardness, thus their psycho- 
logical pressure resistance and resilience are better than undergraduates. Therefore, nursing students should carry out self-psychological health education, improve their psychological regulation ability, and change their roles to grow up healthily in the complex social environment. With regard to different clinical stages, there were significant differences in somatization, compulsion, tenacity and strength $(\mathrm{P}<0.001, \mathrm{P}<0.05)$. Based on clinical practice, it was found that nursing students mainly have problems with somatization, which includes the following main symptoms: dizziness, palpitation and insomnia and other physical discomfort problems. There are no organic problems after relevant examinations, but physical symptoms cannot be alleviated. Upon relevant psychological counseling, it was found that pressure involving internship and employment is the main reason. The body suffered from physical discomfort with benefit finding due to lack of timely discovery and counseling, allowing them to escape the pressure of internship and employment temporarily. However, the nursing students are not aware of this problem, and are still troubled by physical discomfort, unable to go to work normally, and even interrupt their internship. Therefore, both physiological and psychological status of nursing students should be paid more attention to. And they should be taught about how to make psychological adjustment, psychological decompression and how to deal with stress when encountering stress and emotion.

\section{Conclusions}

Guide nursing students to learn to adopt active coping methods to solve life events, relieve the pressure they face in time, and ultimately improve the mental health of nursing students. Strengthen communication with nursing students to ensure that nursing students can better complete clinical internship tasks while the epidemic prevention and control are normalized. At the same time, hospitals should pay more attention to clinical nursing students' mental health and develop prompt intervention programs for nursing interns' psychological crises in response to public health catastrophes. Encourage nursing students to recognize their worth in the workplace and to not feel bad if they make a minor error. Greater positive thinking and the removal of negative emotions, as well as more communication and sharing with classmates and friends, should be done in terms of psychological adjustment, self-discovery, self-dialogue, and self-encouragement. When the situation is serious, you can also seek the support of professional psychological counseling or psychotherapy institutions. It is vital to improving communication among family members in terms of family relations. In the face of the epidemic's hard test, all sectors of society must actively fulfill their social responsibilities in the new period and become a constructive force in the epidemic's prevention and control. For example, social news media should actively promote favorable public opinion and strong advice, aggressively report positive epidemic information, and improve the propaganda of epidemic preventive knowledge. Simultaneously, it is vital to develop self-media regulation and over- 
sight, debunk rumors quickly, and improve information veracity. Psychological assistance services for the majority of nursing students in need should be actively provided by social mental health medical institutions, and psychological puzzles and contradictions should be resolved based on the psychological characteristics and potential psychological problems of clinical nursing students.

There are definitely some psychological problems among clinical nursing students during the pandemic prevention and control. In the "post-pandemic era", the pandemic gradually alleviated, so teaching groups in hospitals should pay more attention to the psychological health of nursing students. It is suggested that hospitals should set up psychological relief groups, and establish various communication and decompression rooms to provide targeted psychological care and counseling to nursing students. Meanwhile, communication with nursing students should be increased to ensure that a better completion of clinical internship tasks while normalizing pandemic prevention and control.

\section{Conflicts of Interest}

The authors declare no conflicts of interest regarding the publication of this paper.

\section{References}

[1] Wang, Z.Q., Zhu, Z.H., Zhu, H.W., et al. (2003) Psychological Crisis Intervention Model in Xiaotangshan Hospital of PLA. Chinese Mental Health Journal, 17, 587-590.

[2] Zhao, G.Q., Wang, Y.G., Wang, Y.Q., et al. (2009) Psychological Crisis Intervention in Disasters: Comment on Psychiatric View. Advances in Psychological Science, 17, 489-494.

[3] Zhang, W.R., Wang, K., Yin, L., Zhao, W., Xue, Q., et al. (2020) Mental Health and Psychosocial Problems of Medical Health Workers during the COVID-19 Epidemic in China. Psychother Psychosom, 89, 242-250. https://doi.org/10.1159/000507639

[4] Ran, M.S., Gao, R., Lin, J.X., Zhang, T.M., et al. (2020) The Impacts of COVID-19 Outbreak on Psychological Health in General Population in Different Areas in China. Psychological Medicine, 51, 1-10.

https://doi.org/10.1017/S0033291720004717

[5] Ministry of Education (2020) Better Prevention and Control of COVID-19 via the Deployment Education System of the Ministry of Education. (In Chinese) http://www.moe.gov.cn/jyb_xwfb/gzdt_gzdt/s5987/202001/t20200122_416316.html

[6] Zhang, F.Y., Li, S.Y., Li, L.L., Cui, J.B., Tian, Y.L., et al. (2020) Emergency Management in University Students during Coronavirus Disease 2019 Epidemics: West China Urgent Recommendation. Chinese Journal of Evidence-Based Medicine, 1-6. https://kns.cnki.net/kcms/detail/51.1656.r.20200224.1026.002.html

[7] Wu, M., Han, Y.J., Sun, X.Q., et al. (2019) The Mediating Effect of Mental Resilience on Stress and Subjective Well-Being of Undergraduate Nursing Students. Occupational and Health, 35, 250-253.

[8] Wang, X.D., Wang, X.L. and Ma, H. (1999) Manual of Mental Health Rating Scale. Revised and Enlarged Edition, Chinese Mental Health Journal, Beijing, 113-115.

[9] Shi, L.Y., Huang, S.P., Zhuo, L., et al. (2013) Reference Value, Reliability and Validity Evaluation of SCL-90 Scale for College Students. Chinese Journal of School Health, 
34, 223-224.

[10] Yu, X. and Zhang, J. (2007) Factor Analysis and Psychometric Evaluation of the Connor-Davidson Resilience Scale (CDRISC) with Chinese People. Social Behavior Personality: An International Journal, 35, 19-30.

https://doi.org/10.2224/sbp.2007.35.1.19

[11] Yu, X.N. and Zhang, J.X. (2007) A Comparison between the Chinese Version of Ego-Resiliency Scale and Connor-Davidson Resilience Scale. Journal of Psychological Science, 5, 1169-1171.

[12] Connor, K.M. and Davidson, J.R.T. (2003) Development of a New Resilience Scale: The Connor-Davidson Resilience Scale (CDRISC). Depress Anxiety, 18, 76-82. https://doi.org/10.1002/da.10113

[13] Xin, Z.Q. and Chi, L.P. (2020) Changing Trend of Contemporary Chinese Psychological Health. People's Tribune, 29, 46-50.

[14] Liu, Y.Y. (2018) Analysis of Mental Health of Chinese Population and Their 30-Year Changes Based on SCL-90 Scale. Master's Thesis, The Fourth Military Medical University, Xi'an. 